\title{
Erratum to: Isolation and fine mapping of Rps6: an intermediate host resistance gene in barley to wheat stripe rust
}

\author{
Andrew M. Dawson ${ }^{1} \cdot$ John N. Ferguson ${ }^{1,2} \cdot$ Matthew Gardiner $^{1} \cdot$ Phon Green $^{1}$ \\ Amelia Hubbard $^{3} \cdot$ Matthew J. Moscou $^{1}$
}

Published online: 1 March 2016

(C) Springer-Verlag Berlin Heidelberg 2016

\section{Erratum to: Theor Appl Genet DOI 10.1007/s00122-015-2659-x}

Unfortunately, the articles 10.1007/s00122-015-2659-x (Isolation and fine mapping of Rps6: an intermediate host resistance gene in barley to wheat stripe rust) and 10.1007/s00122-015-2663-1 (Fine mapping of barley locus Rps6 conferring resistance to wheat stripe rust) were accepted and published as side-by-side coordinated publications. However, because of a communication problem the online publication of article 10.1007/s00122-0152663-1 was published later than the related article 10.1007/ s00122-015-2659-x.

The online version of the original article can be found under doi:10.1007/s00122-015-2659-x and doi:10.1007/s00122-0152663-1.

Matthew J. Moscou

matthew.moscou@sainsbury-laboratory.ac.uk

1 The Sainsbury Laboratory, Norwich Research Park, Norwich NR4 7UH, UK

2 Present Address: School of Biological Sciences, University of Essex, Colchester CO4 3SQ, UK

3 National Institute of Agricultural Botany, Huntingdon Road, Cambridge CB3 OLE, UK 